\title{
Aspectos biológicos del pleco invasor Pterygoplichthys pardalis (Teleostei: Loricariidae) en el río Palizada, Campeche, México
}

\section{Biological aspects of the invasor plecos Pterygoplichthys pardalis (Teleostei: Loricariidae) from Palizada River, Campeche, Mexico}

\author{
Armando T. Wakida-Kusunoki ${ }^{1 *}$ y Luis Enrique Amador-del Ángel ${ }^{2}$ \\ ${ }^{1}$ Instituto Nacional de Pesca, Centro Regional de Investigación Pesquera. Av. Héroes del 21 de Abril s/n, Col. Playa Norte, 24120 Ciudad del Carmen, \\ Campeche. México. \\ ${ }^{2}$ Centro de Investigación de Ciencias Ambientales (CICA), Universidad Autónoma del Carmen. Av. Laguna de Términos s/n, Col. Renovación 2 da \\ sección, 24155 Ciudad del Carmen, Campeche, México. \\ *Correspondencia: armandowakida@yahoo.com.mx
}

\begin{abstract}
Resumen. Se analizó el comportamiento de la abundancia, la relación peso-longitud y el índice gonadosomático de Pterygoplichthys pardalis en el río Palizada en Campeche, México. Se realizaron muestreos mensuales de junio de 2008 a mayo de 2009 con redes agalleras. Se colectaron 118 plecos cuyas tallas estuvieron entre 222 y $422.5 \mathrm{~mm}$ de longitud total (LT) y un peso de 72.8 a 385.4 g. El periodo de mayor abundancia se presentó de junio a agosto, no hubo diferencias significativas de la abundancia en diferentes periodos del día $(p \leq 0.05)$. Se observó que el índice de abundancia está correlacionado con el nivel del río y el índice gonadosomático. El índice gonadosomático muestra que la época de reproducción se presenta de mayo a agosto; sin embargo, se encontraron hembras con gónadas bien desarrolladas durante todo el año de muestreo. Los parámetros de la relación peso-longitud para ambos sexos son: $\mathrm{b}=$ 2.78 y a $=0.0003$. La comparación de esta relación entre ambos sexos no resultó significativa $(p \leq 0.05)$. Es necesario realizar esfuerzos para la erradicación de este pez por medio de incentivos a pescadores durante el periodo de mayor vulnerabilidad y de mayor actividad reproductiva.
\end{abstract}

Palabras clave: Pterygoplichthys pardalis, época reproductiva, relación peso longitud, índice gonado-somático, río Palizada, México.

\begin{abstract}
Abundance, length-weight relationship and gonad somatic index of Pterygoplichthys pardalis in Palizada River, Mexico were analyzed. Monthly samplings were carried out from June 2008 to May 2009 with gill nets. A total of 118 plecos were catched; theirs sizes were between 222 and $422.5 \mathrm{~mm}$ of total longitude (TL) and weight between 72.8 to $385.4 \mathrm{~g}$. The highest abundance period was found from June to August, with not statistical differences between their values in the different periods of the day $(p \leq 0.05)$. The abundance index was correlationed with river level and gonad somatic index. The gonad somatic index showed that the spawning season occurs from May to August; however, females with well developed gonads were observed during all sampling period. The parameters of the length-weight relationship for both sexes are: $b=2.78$ and $a=0.0003$. The comparison of this relationship between both sexes is not significant $(p \leq 0.05)$. It is necessary that efforts be made to eradicate this species through incentives to fishermen during bigger vulnerability and of more reproductive activity period.
\end{abstract}

Key words: Pterygoplichthys pardalis, reproductive season, length-weight relationship, gonad somatic index, Palizada River, México

\section{Introducción}

El pleco Pterygoplichthys pardalis es nativo de las partes bajas, medias y altas de la cuenca de río Amazonas (Weber, 2003). Esta especie se ha introducido en varias regiones del mundo, como Singapur (Tan y Tan, 2003), Filipinas (Chavez et al., 2006), Indonesia, Malasia (Page y

Recibido: 08 marzo 2010; aceptado: 24 enero 2011
Robins, 2006; Samat et al., 2008) y Vietnam (Levin et al., 2008). En México fue registrada por primera vez en 2007 en el río Grijalva (Wakida-Kusunoki et al., 2007); desde esa fecha, la especie se ha expandido a otras regiones del país, como es el caso de Campeche (Wakida-Kusunoki y Amador-del Ángel, 2008).

De acuerdo con Mendoza et al. (2007) estos organismos se consideran una de las mayores amenazas a la biodiversidad de los ecosistemas continentales en México y sus pesquerías de agua dulce. El estudio de los aspectos 
biológicos y las características poblacionales de las especies exóticas en los nuevos hábitats son indispensables para su manejo. En el caso del género Pterygoplichthys, sólo se han realizado estudios acerca de su reproducción en Taiwán y Florida (Liang et al., 2005; Gibss et al., 2008) y de relaciones morfológicas en Malasia (Samat et al., 2008)

El presente trabajo tiene como objetivo conocer algunos aspectos biológicos que sirvan para el control y erradicación del pleco $P$. pardalis en el río Palizada, Campeche, México. Se presentan los resultados de la abundancia, la relación peso-longitud, el periodo reproductivo y la proporción de sexos.

\section{Materiales y métodos}

El río Palizada pertenece a la cuenca del GrijalvaUsumacinta, que es una de las cuencas más importantes de México; sus escurrimientos representan el 30\% de los ríos de México y sus ecosistemas albergan el 64\% de la biodiversidad nacional conocida (Toledo, 2003). En la zona se han registrado 64 especies de peces (Ramos-Miranda et al., 2006). En esta región hay 3 periodos climáticos: la estación seca (marzo-mayo), el periodo de lluvias (junioseptiembre) y el de nortes (octubre-febrero).

Para la realización de este trabajo se efectuaron muestreos mensuales de junio de 2008 a mayo de 2009 en la porción baja del río Palizada (Fig. 1). La recolección de los peces se llevó a cabo mediante 3 redes agalleras de monofilamento de nylon de $100 \mathrm{~m}$ de longitud por 1 $\mathrm{m}$ de altura, con una luz de malla de $7.6 \mathrm{~cm}$. Cada una fue colocada en una de 3 estaciones localizadas en los bordes del río debido a la fuerte corriente existente y a su profundidad. Las redes fueron revisadas cada 2 horas entre las 14:00 y las 21:00. La vegetación circundante a los bordes del río es predominantemente de sauces, Salix chilensis y zarzales, Mimosa pigra, así como pastos introducidos para la ganadería (Guevara-Carrió et al., 2009). En cada muestreo, se registró la temperatura y la salinidad con apoyo de una sonda multiparámetros YSI modelo 63. La información del nivel del río fue proporcionada por la Comisión Nacional del Agua (CNA).

Los organismos capturados fueron transportados en hielo al laboratorio, donde se midió la longitud total (LT) de cada ejemplar con la ayuda de un ictiómetro $( \pm 0.5 \mathrm{~mm})$. El peso total (PT) y el peso de la gónada se obtuvieron con una balanza digital de $0.01 \mathrm{~g}$ de precisión. El sexo fue determinado por observación directa de las gónadas.

La abundancia de plecos en la zona se analizó por medio de la captura por unidad de esfuerzo (CPUE), que consistió en número de organismos capturados entre la duración de las redes en el agua. Los valores de la CPUE se compararon en diferentes periodos del día para determinar si había diferencias de capturabilidad. Estos periodos se determinaron dependiendo de la cantidad de luz solar existente y se denominaron: día, de 2 a 5 pm; crepuscular, de 5 a 7 pm, y nocturno, de 7 a 9 pm. Para la comparación entre meses y periodos del día, se utilizó el análisis de varianza de una vía con significancia $p<0.05$ (Zar, 1996).

La talla de primera madurez $\left(\mathrm{L}_{50}\right)$ se determinó considerando sólo las hembras maduras, siguiendo el criterio de Nikolski (1963), con gónadas bien desarrolladas con presencia de huevos. $\mathrm{La}_{50}$ se estimó mediante el ajuste de una curva logística a partir de los datos de frecuencia relativa acumulada de las hembras maduras. El ajuste se realizó mediante la fórmula

$$
\text { frec. rel. }=\frac{c}{a+\exp \left(b^{*} L T\right)}
$$

donde $a, b$ y $c$ son parámetros del ajuste, frec. rel, es la frecuencia relativa acumulada de hembras maduras y $L T$ es la longitud total (Santana-Hernández et al., 1996).

Para la determinación del índice gonadosomático (IGS) se utilizaron las gónadas de las hembras por ser más fáciles de identificar debido al tamaño de los huevos (Liang et al., 2005). Para el cálculo de IGS se utilizó la siguiente fórmula:

$$
I G S=\frac{P G}{P T} * 100
$$

donde PG es peso de la gónada y PT es peso total del organismo. Se espera que el valor de IGS se incremente en una hembra cuando se presenta el periodo de reproducción y alcance su máximo valor, justo antes del desove (Hoggarth et al., 1999; Liang et al., 2005; Arias et al., 2006). Para comparar los valores del IGS entre meses se utilizó un análisis de varianza de una vía (Zar, 1996).

Para conocer el comportamiento de las variables ambientales se realizó un análisis exploratorio de datos que consistió en la construcción de gráficas (Du Toit et al., 1986). Además para saber si existía relación entre la abundancia y el IGS con las variables ambientales se realizó un análisis de correlación y pruebas de significancia con el valor del coeficiente de correlación (Zar, 1996; Weimer, 1996).

La relación peso total-longitud total (PT-LT) para machos y hembras se calculó utilizando la regresión potencial

$$
\mathrm{PT}=a \mathrm{LT}^{b}
$$

donde $a$ es una constante de regresión equivalente al factor de condición y $b$ el coeficiente de crecimiento de la regresión (Ricker, 1975). Para confirmar si los valores de $b$ obtenidos en las regresiones lineales eran 

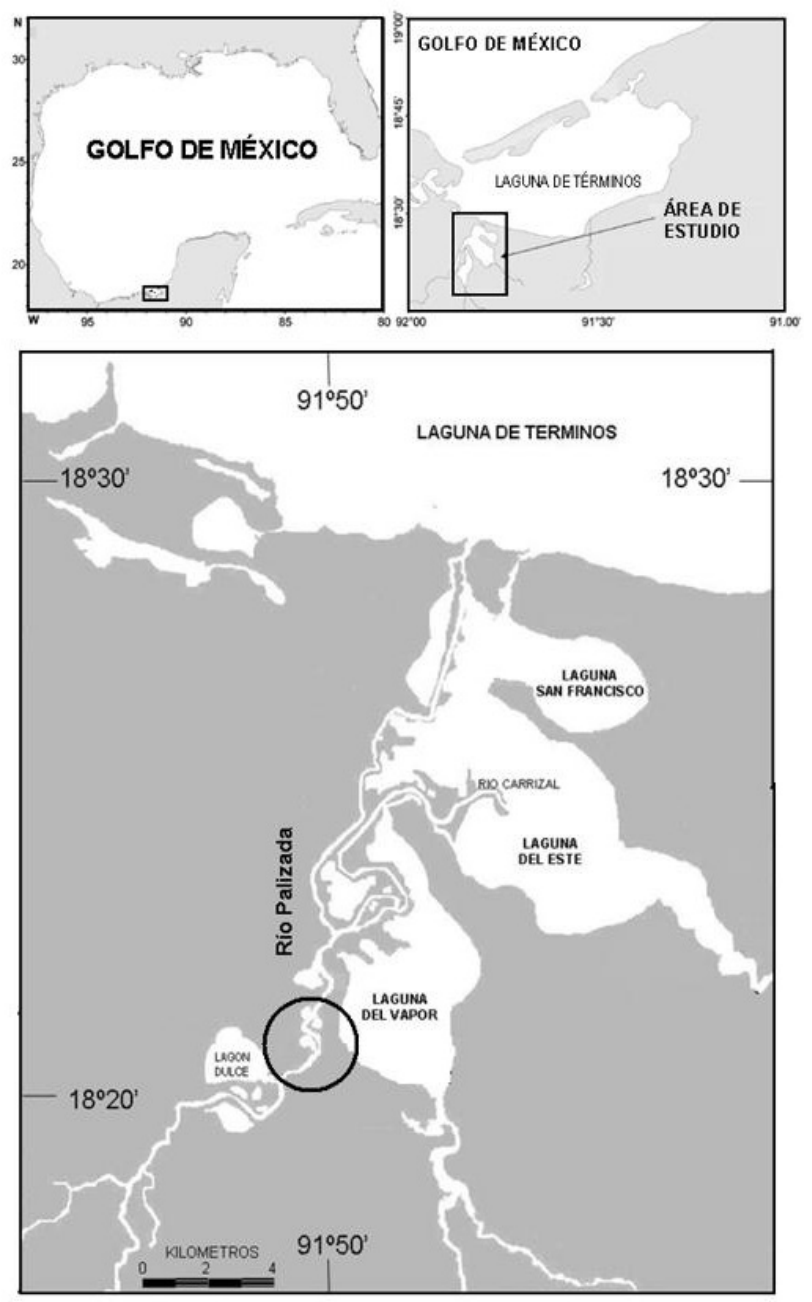

Figura 1. Área de estudio. El círculo señala el área donde se realizaron los muestreos.

significativamente diferentes al valor de isometría (3) se realizó una prueba t-Student (Sokal y Rohlf, 1987). Los valores menores y mayores a 3 indican un crecimiento alométrico negativo y positivo, lo que indica que un pez no cambia mucho sus proporciones con el crecimiento (Pauly, 1984).

Para conocer si existían diferencias entre la relación peso-longitud entre sexos se llevó a cabo una prueba de comparación de pendientes de las regresiones lineales realizadas (Zar, 1996).

\section{Resultados}

Se capturaron 277 organismos; de los cuales, el $41.16 \%$ (118 peces, alrededor de 9.8 organismos por mes) fueron $P$. pardalis. En peso, el porcentaje de P. pardalis con respecto al total fue de $32.57 \%$. La captura incidental estuvo compuesta por 19 especies, de las cuales las más importantes en número fueron: arenga, Dorosoma anale (17.9\%); sábalo, Megalops atlanticus (10.8\%); bandera, Bagre marinus (6.86\%), y los bagres Ictalurus meridionalis (5.05\%) y Cathorops aguadulce (4.33\%).

La talla de P. pardalis fue de 222 a $422.5 \mathrm{~mm}$ de longitud total (LT) y el peso varió de 72.8 a 385.4 gramos. En el Cuadro 1 se muestran las diferentes medidas corporales de $P$. pardalis obtenidas en el presente trabajo.

El índice de abundancia calculado indica que en julio se presentaron los valores significativamente más altos del periodo de muestreo (Fig. 2, Cuadro 2). La CPUE de plecos presentó los valores más altos en las horas nocturnas (Fig. 3). Aunque no varió significativamente durante los periodos del día muestreados (Cuadro 2).

En cuanto a los aspectos reproductivos, la talla promedio de primera madurez gonádica de las hembras se encontró alrededor de los 306.8 mm LT (Fig. 4). Los valores del IGS presentan su máximo en julio y los mínimos en octubre y febrero (Fig. 5).

Por lo que se refiere a los parámetros ambientales, el nivel del río presentó valores altos de julio a noviembre, como consecuencia de la época de lluvias y nortes. Los valores bajos se registraron en el resto de los meses (Fig. 6a). La temperatura presentó sus mayores valores durante agosto, septiembre y octubre y los menores de noviembre a enero (Fig. 6b). La salinidad (UPS) presentó valores oligohalinos de $0.2 \pm 0.1$; los más bajos, durante la época de lluvias y nortes y los mayores en época de secas (febrero a mayo) (Fig. 6c).

El análisis de correlación muestra que entre la abundancia y el nivel del río la relación fue significativa y positiva $(p \leq 0.05)$, al igual que entre la abundancia y el IGS (Cuadro 3 ).

Los valores de la relación exponencial peso-longitud para los organismos de $P$. pardalis se muestran en el Cuadro 4. Los coeficientes de regresión, tanto de hembras como de machos fueron significativamente menores a 3 , lo cual indica que esta especie presenta un crecimiento

Cuadro 1. Valor de las diferentes medidas corporales (promedio \pm desviación estándar) de $P$. pardalis, en el río Palizada, México

\begin{tabular}{lccccc}
\hline & $n$ & $L T(\mathrm{~mm})$ & $L F(\mathrm{~mm})$ & $L P(\mathrm{~mm})$ & $P T(\mathrm{~g})$ \\
\hline Machos & 55 & $299.82 \pm 31.07$ & $258.79 \pm 30.26$ & $225.01 \pm 28.25$ & $223.25 \pm 61.84$ \\
Hembras & 59 & $297.44 \pm 44.17$ & $252.99 \pm 38.81$ & $218.55 \pm 37.09$ & $198.90 \pm 74.54$ \\
Total & 114 & $298.71 \pm 37.59$ & $256.09 \pm 34.48$ & $222.00 \pm 32.69$ & $211.90 \pm 68.84$ \\
\hline
\end{tabular}


Cuadro 2. Resultados de los análisis de varianza para abundancia, índice gonadosomático y abundancia durante los periodos del día de $P$. pardalis en el río Palizada, México

\begin{tabular}{lcccc}
\hline \multicolumn{1}{c}{ Fuente } & $\begin{array}{c}\text { Grados de } \\
\text { libertad }\end{array}$ & $\begin{array}{c}\text { Sumade } \\
\text { cuadrados }\end{array}$ & $F$ & Probabilidad \\
\hline Abundancia & & & & \\
$\begin{array}{l}\text { Entre } \\
\text { meses }\end{array}$ & 10 & 9.12 & 3.463 & 0.0099 \\
$\begin{array}{l}\text { Dentro de } \\
\text { meses }\end{array}$ & 22 & 5.85 & & \\
$\begin{array}{l}\text { Índice } \\
\text { Gonadosomático }\end{array}$ & 10 & 269.429 & 6.252 & 0.00032 \\
$\begin{array}{l}\text { Entre } \\
\text { meses }\end{array}$ & 22 & 95.756 & & \\
$\begin{array}{l}\text { Dentro de } \\
\text { meses }\end{array}$ & & & \\
$\begin{array}{l}\text { Comportamiento de la abundancia } \\
\begin{array}{l}\text { Entre } \\
\text { periodo* }\end{array}\end{array}$ & 2 & 1.14 & 0.075 & 0.928 \\
$\begin{array}{l}\text { Dentro } \\
\text { periodo }\end{array}$ & 30 & 28.255 & & \\
\hline
\end{tabular}

* Periodo: diurno, nocturno y crepuscular.

alométrico negativo (Tesch, 1971). La comparación entre las pendientes de las regresiones no resultaron con diferencia significativa $(p<0.05)$.

\section{Discusión}

El alto porcentaje que se encontró de $P$. pardalis en la captura total de los organismos capturados, nos podría indicar el grado de presencia que tiene esta especie en los ecosistemas acuáticos a los cuales invade.

El índice de abundancia está correlacionado significativamente con el nivel del río, lo que coincide con otros autores que mencionan que la capturabilidad en ríos y lagunas se afecta por el volumen de agua presente, que está determinado por los flujos estacionales (Hoggarth et al., 1999; García et al., 2003; Jones, 2003; Luzhnyak y Korneev, 2006). Los niveles más altos del agua incrementan el tamaño del ambiente acuático y de nutrientes que estimulan
Cuadro 4. Valor de los parámetros de la relación PT-LT de $P$. pardalis, en el río Palizada, México

\begin{tabular}{lcccc}
\hline & $n$ & $a$ & $b$ & $r^{2}$ \\
\hline Machos & 63 & 0.00004 & $2.7209^{*}$ & 0.9286 \\
Hembras & 55 & 0.00002 & $2.8392^{*}$ & 0.8782 \\
Total & 118 & 0.00003 & $2.7819^{*}$ & 0.8921 \\
\hline
\end{tabular}

*Significativamente diferente a $3(p \leq 0.05)$.

el rápido crecimiento de micro-organismos, invertebrados y plantas, proporcionando comida abundante (LoweMcConnell, 1975, 1987; Saint-Paul et al., 2000; Rahim et al., 2009). Esta situación podría explicar los valores altos de CPUE en junio y julio, pero no para los meses subsecuentes, donde también se presentaron valores altos de niveles de agua, lo que indica que existen otros factores que podrían estar incidiendo en la capturabilidad en esos meses.

Welcomme $(1979,1987)$ observa que durante los niveles bajos de los ríos la mayoría de los peces tienden a permanecer estáticos; lo cual coincide con los resultados obtenidos en este trabajo, que muestran los menores valores de abundancia en los meses cuando los niveles fueron más bajos.

En otro caso, el índice de abundancia presentó correlación significativa con los valores de IGS, debido a que durante el periodo de mayor actividad reproductiva estos organismos son más activos, lo cual tiene como consecuencia un aumento en su captura, y por ende un incremento del CPUE. El aumento en la actividad como efecto del comportamiento reproductivo se ha observado en otras especies (Miller, 1963; Ellis y Giles, 1965, Ribeiro, 1983; Fernandes, 1997). Según Granado-Lorencio et al. (2005), P. pardalis es una especie sedentaria que se captura principalmente cuando es madura (Neves y Rufino, 1998; Cerdeira y Ruffino, 2000).

Los valores del CPUE no mostraron diferencias estadísticas significativas en los diversos periodos del día. Los resultados gráficos muestran valores superiores durante los periodos nocturnos. Al respecto, la mayoría de autores considera que en general las especies de la familia Locariidae son de hábitos nocturnos (Power, 1984a; Hahn et al. 1997; Casatti y Castro, 1998; Mendoza et al., 2009). Tal vez la alta concentración de sedimentos en suspensión que presenta el

Cuadro 3. Valor de las correlaciones entre la abundancia y el IGS de P. pardalis y las variables ambientales en el río Palizada, México

\begin{tabular}{lcccc}
\hline & Abundancia & IGS & Nivel del río $(m$ snm $)$ & Temperatura $\left({ }^{\circ} \mathrm{C}\right)$ \\
\hline Índice Gonadosomático & $0.860^{*}$ & & & \\
Nivel de río & $0.524^{*}$ & 0.152 & 0.380 & -0.353 \\
Temperatura & 0.456 & 0.424 & $-0.740^{*}$ & -0.043 \\
Salinidad (UPS) & -0.249 & -0.35 & \\
\hline
\end{tabular}

* Correlación significantiva. 


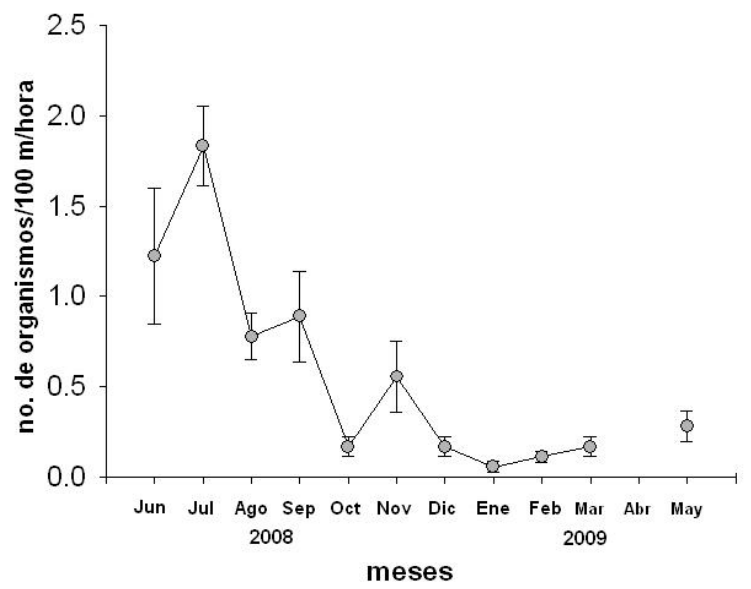

Figura 2. Comportamiento mensual de la abundancia (organis$\mathrm{mos} / 100 \mathrm{~m}$ de red/hora) de P. pardalis en el río Palizada, México.

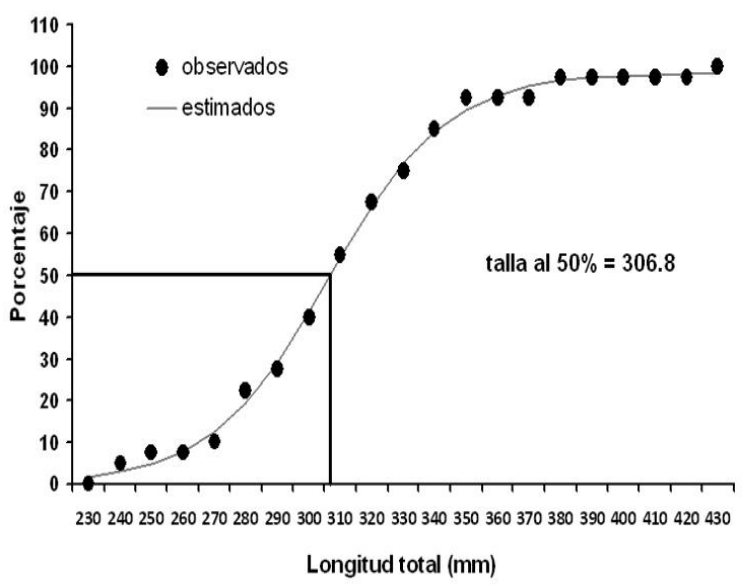

Figura 4. Talla promedio de primera madurez de $P$. pardalis en el río Palizada, México.

río Palizada (Páez-Osuna et al., 1987; Rendón-Von Osten et al., 2005) tenga como resultado que estos organismos presenten mayor actividad durante el día. Schulz y Leuchtenberger (2006) mencionan que la turbidez puede provocar un cambio de comportamiento nocturno a diurno en peces gato. Tal condición se ha observado en juveniles de Loricariichthys anus que durante el día estuvieron más activos que en la noche en aguas de alta turbidez del lago Dos Quadros en el sur de Brasil (Petry y Schulz, 2000).

Los resultados de abundancia de plecos podrían considerarse bajos, lo cual se debe a que el río Palizada presenta en su canal principal aguas con una alta concentración de sedimento en suspensión. Se ha indicado que las especies de plecos prefieren aguas claras y someras (Power, 1984b), por lo que es probable que esta especie

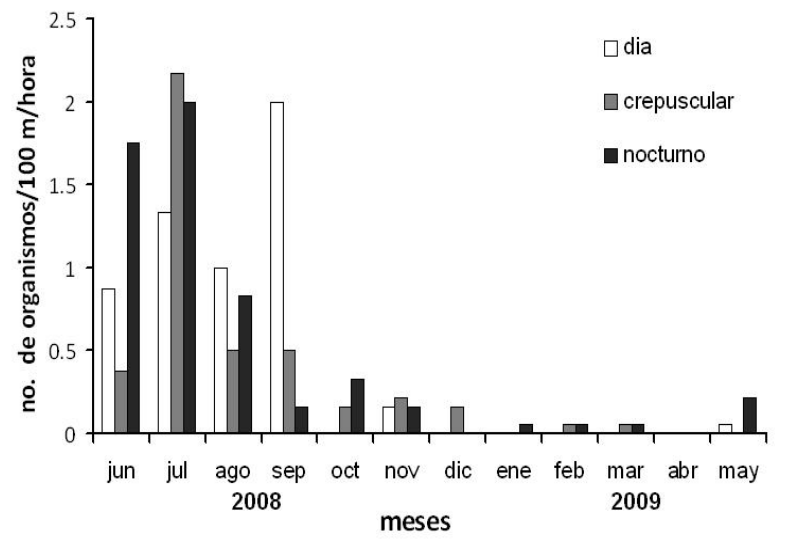

Figura 3. Comportamiento de la abundancia (organismos/100 m de red/hora) de $P$. pardalis en los diferentes periodos del día en el río Palizada, México.

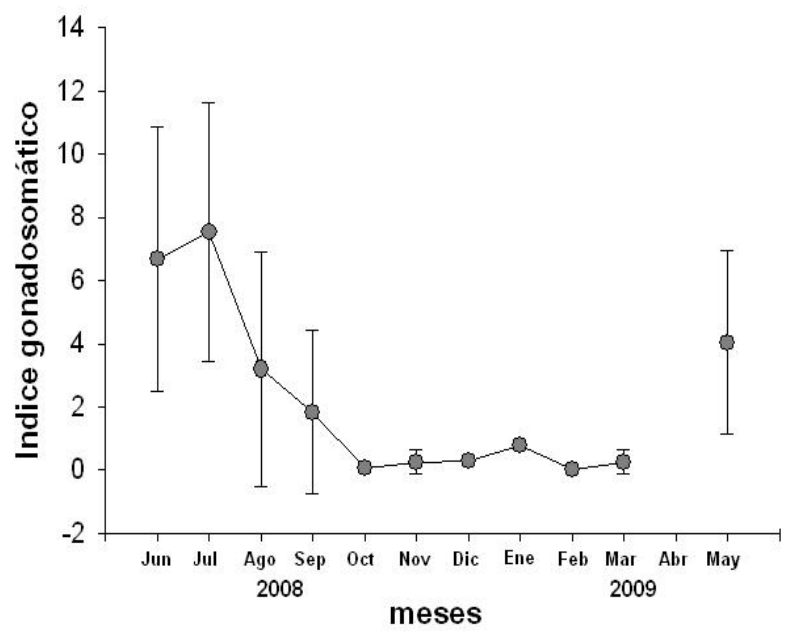

Figura 5. Comportamiento del índice gonadosomático de $P$. pardalis en el río Palizada, México.

se extienda a lagunas subyacentes de la cuenca, donde se encuentran esas condiciones.

El comportamiento de los valores del IGS es muy similar a lo que se ha encontrado en otras regiones del mundo donde se han presentado invasiones con especies cercanas a $P$. pardalis. Liang et al. (2005) encontraron en Taiwán que los mayores valores del IGS de $P$. multiradiatus se presentaron de mayo a septiembre, con máximos en junio y julio. Para $P$. disjunctivus en Florida, se determinó que el periodo con valores mayores se presenta de abril a septiembre (Gestring et al., 2006). Este comportamiento parece estar relacionado con la época de lluvias, ya que en esas regiones, al igual que en el río Palizada, la época de lluvia coincide con la época en la que los valores de IGS son mayores. Lowe-McConnell (1975) y Winemiller 

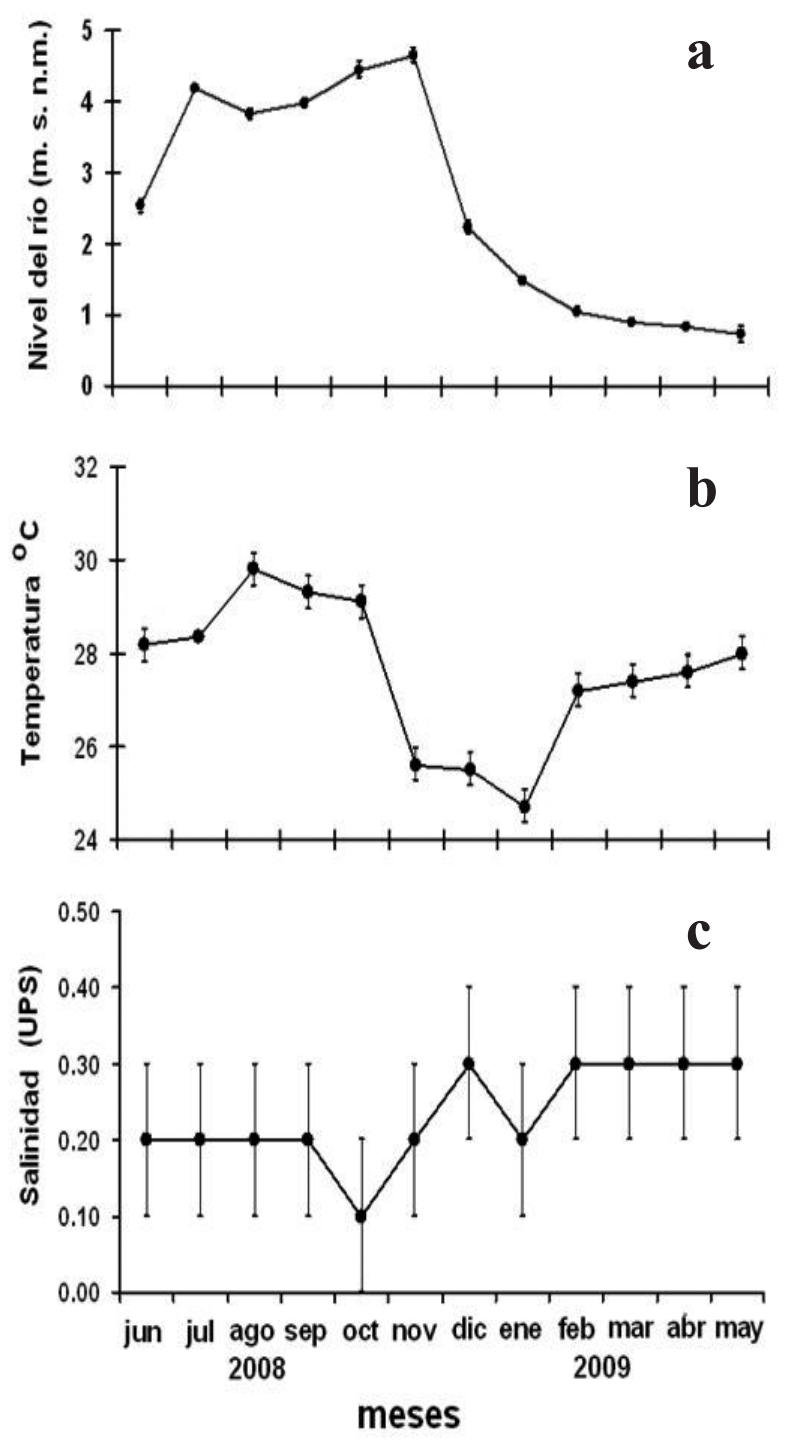

Figura 6. Comportamiento de los parámetros ambientales en el río Palizada durante el periodo de muestreo: a, nivel del río (m snm); b, temperatura $\left({ }^{\circ} \mathrm{C}\right)$ y c, salinidad (UPS).

(1987) mencionan que muchos de los peces ostariofisos en zonas tropicales presentan una relación entre su periodo de reproducción y los flujos de las primeras lluvias fuertes. Duarte y Araújo (2001) y Duarte et al., (2007) atribuyeron este patrón de abundancia de Loricariichthys spixii en el reservorio de Lajes, Brasil al incremento en la disponibilidad de los recursos alimenticios. El valor de la talla promedio de primera madurez depende en gran medida de las tallas capturadas, en este estudio el mayor porcentaje de hembras maduras se encontró entre 280 y $340 \mathrm{~mm}$ LT, lo que coincide con lo presentado con Liang et al. (2005) para P. multiradiatus donde la mayoría de los valores altos del IGS los encontró en ese rango de tallas. La disminución tanto de la abundancia como del IGS podría ser resultado del aumento de aproximadamente un metro en el nivel del río que se presentó en octubre y provocó el desbordamiento. Esta situación pudo haber propiciado una dispersión de los organismos en la zona.

Como se mencionó anteriormente, es probable que los plecos se dispersen hacia lagunas, las cuales se consideran como reservorios de una gran diversidad de plantas acuáticas (Ocaña y Lot, 1996). Estas áreas son de mucha importancia para el desarrollo de numerosas especies de peces y otros organismos, las cuales las utilizan como sitios de crianza y alimentación de larvas y juveniles. Se espera que un aumento o dispersión de los plecos en la zona, tenga como resultado un efecto negativo sobre las poblaciones de estas especies, ya que se conoce que un pequeño número de plecos son capaces de reducir de manera considerable la abundancia de plantas acuáticas (Power, 1990). Por lo anterior, es necesario que se realicen acciones para controlar la expansión de esta especie. Se podrían proponer planes de erradicación mediante incentivos. El esfuerzo mayor deberá realizarse de mayo a agosto para que la captura se efectúe en un periodo sensible de la especie, como lo es la época de reproducción, estimada en este trabajo. Otra opción sería incentivar el consumo de la especie, puesto que $P$. pardalis ocupa un lugar importante en las capturas comerciales en el área del Amazonas (Cerdeiras y Ruffino, 2000).

En cuanto a los valores considerados para calcular la relación peso-longitud, se encontró que en todos los organismos capturados durante el periodo de estudio, el parámetro "b" está en el intervalo de valores que se han estimado para este tipo de organismos (Liang et al., 2005; Samat et al., 2008). Los valores fueron menores a 3, lo que corresponde a un crecimiento alométrico negativo que indica que los individuos de $P$. pardalis empiezan a ser más delgados conforme crecen (Samat et al., 2008). Las diferencias o similitudes respecto al valor de "b" además de ser atribuidas a factores intrínsecos (genéticos) de las especies, se relacionan también con el hábitat y la época del año (Gómez G. y R. Guzmán, 1998). Desafortunadamente, el número de organismos capturados no permitió evaluar los cambios temporales en los parámetros de la relación peso-longitud. La comparación entre las pendientes del análisis de regresión no muestra diferencias significativas, lo cual no indica que presenten el mismo el tipo de crecimiento.

\section{Agradecimientos}

A Isaac Rojas González por los comentarios al trabajo. A Juan Sánchez Pérez y Pablo Laffón Leal por su apoyo en trabajo de campo. 


\section{Literatura citada}

Arias, C. J. A., E. Zaniboni-Filho y B. E. Aya. 2006. Indicadores del ciclo reproductivo del yamú Brycon amazonicus en cautiverio. Orinoquia - Universidad de los Llanos 10:24-34.

Casatti, L. y R. M. C. Castro. 1998. A fish community of the São Francisco River headwaters riffles, southeastern Brazil. Ichthyological Exploration of Freshwaters 9:229-242.

Cerdeira, R. P. G. y M. L. Ruffino. 2000. Fishes catch among Riverside communities around Lago Grande de Monte Alegre, lower Amazonas, Brasil. Fisheries Management and Ecology 7:355-374.

Chávez, J. M., R. M. de la Paz, S. K. Manohar, R. C. Pagulayan y J. R. Carandang. 2006. New Philippine record of South American sailfin catfishes (Pisces: Locariidae). Zootaxa 1109:57-68.

Duarte, S. y F. G. Araújo. 2001. Abundância relativa e distribuição de Loricariichthys spixii (Siluriformes, Loricariidae) no reservatório de Lajes, Rio de Janeiro, Brasil. Revista Brasileira de Zoologia 18:465-477.

Duarte S., F. G. Araújo, A. Sales y N. Bazzoli. 2007. Morphology of gonads, maturity and spawning season of Loricariichthys spixii in a subtropical reservoir. Brazilian Archives of Biology and Technology 50:1019-1032.

Du Toit, S. H. C., A. G. W. Steyn y R. H. Stumpf. 1986. Graphical exploratory data analysis. Springer, New York. $314 \mathrm{p}$.

Ellis, D. V. y M. A. Giles. 1965. The spawning behavior of the walleye, Stizostedion vitreum (Mitchill). Transactions of the American Fisheries Society 94:358-362.

Fernandes, C. C. 1997. Lateral migration of fishes in Amazon floodplains. Ecology of Freshwater Fish 6:36-44.

García, A. M., M. B. Raseira, J. P. Vieira, K. O. Winemiller y A. M. Grimm. 2003. Spatio temporal variation in shallowwater freshwater fish distribution and abundance in a large subtropical coastal lagoon. Environmental Biology of Fishes 68:215-228.

Granado-Lorencio, C., C. R. M. Araujo-Lima y J. Lobón-Cerviá. 2005.Abundance-distribution relationships in fish assembly of the Amazonas floodplainlakes. Ecography 28:515-520.

Gestring, K., P. Shafland y S. Murray. 2006. The status of loricariid catfishes in Florida with emphasis on sailfin catfish (Pterygoplichthys multiradiatus). ESA International Conference. Mérida, Mexico 8-12 January 2006 http://abstracts.co.allenpress.com/pweb/esai2006/ document/59128; 22.X.2010.

Gibbs, M. A., J. H. Shields, D. W. Lock, K. M. Talmadge y T. M. Farrell. 2008. Reproduction in an invasive exotic catfish Pterygoplichthys disjunctivus in Volusia Blue Spring, Florida, U.S.A. Journal of Fish Biology 73:1562-1572.

Gómez G. y R. Guzmán. 1998. Relación longitud peso y talla de madurez de la petota (Umbrina coroides), en el norte del estado de Sucre, Venezuela. Zootecnia Tropical 16:267-276.

Guevara-Carrió, E., L. E. Amador-del Ángel y R. Brito-Pérez. 2009. Primer informe parcial del proyecto GN004-005: Aspectos biológicos e impacto socio-económico de los plecos del género Pterygoplichthys y dos ciclídos no nativos en el Sistema Fluvio Lagunar Deltaico río Palizada, en el Área Natural Protegida Laguna de Términos, Campeche. Centro de Investigación de Ciencias AmbientalesUniversidad Autónoma del Carmen/ Comisión Nacional para el Conocimiento y Uso de la Biodiversidad, Ciudad del Carmen, Campeche. 29 p.

Hahn, N. S., V. L. Lescano de Almeida y K. D. Graspar Da Luz. 1997. Alimentação e ciclo alimentar de Hoplosternum littorale (Hankock) (Siluriformes, Callichthyidae) nas lagoas Guaraná e Patos da planície do alto rio Paraná, Brasil. Revista Brasileria de Zoologia 14:57-64.

Hoggarth, D. D., V. J. Cowan, A. S. Halls, M. Aeron-Thomas, J. A. McGregor, C. A. Garaway, A. I. Payne y R. L. Welcomme. 1999. Management guidelines for Asian floodplain river fisheries. Part 2. Summary of DFID research. FAO Fisheries Technical Paper 384/2. FAO, Rome 117 p.

Jones, R. W. 2003. Stock assessment in river systems: brief outline and bibliography. http://www.worldfish.org/PPA/ PDFs/Semi-annual\%20II\%20English/2nd\%20s.a.\%20eng_ E7.pdf; 22.X.2010.

Levin, B. A., P. H. Phuong y D. S. Pavlov. 2008. Discovery of the Amazon sailfin catfish Pterygoplichthys pardalis (Castelnau, 1855) (Teleostei: Loricariidae) in Vietnam. Journal of Applied Ichthyology 24:715-717.

Liang, S. H., H. P. Wu y B. S. Shieh. 2005. Size structure, reproductive phenology, and sex ratio of an exotic armored catfish (Liposarcus multiradiatus) in the Kaoping River of southern Taiwan. Zoological Studies 44:252-259.

Lowe-McConnell, R. H. 1975. Ecological aspect of seasonality in fishes of tropical water. Symposia of the Zoological Society of London 44:219-241.

Lowe-McConnell, R. H. 1987. Ecological studies in tropical fish communities. Cambridge University Press. 400 p.

Luzhnyak, V. A. y A. A. Korneev. 2006. Modern ichthyofauna of the lower Don Basin in conditions of the anthropogenic transformation of its runoff. Journal of Ichthyology 46:525533.

Mendoza, R., S. Contreras, C. Ramírez, P. Koleff, P. Álvarez y V. Aguilar. 2007. Los peces diablo: Especies invasoras de alto impacto. Biodiversitas 70:1-5.

Mendoza, R., E. Alfaro, B. Cudmore, R. Orr, J. P. Fisher, S. Contreras-Balderas, W. R. Courtenay, P. Koleff-Osorio, N. Mandrak, P. Álvarez-Torres, M. Arroyo-Damián, C. Escalera-Gallardo, A. Güevara-Sanguinés, G. Greene, D. Lee, A. Orbe-Mendoza, C. Ramírez-Martínez y O. StabridisArana. 2009. Trinational risk assessment guidelines for aquatic alien invasive species. Test cases for the snakeheads 
(Channidae) and armored catfishes (Loricariidae) in North American inland waters. Commission for Environmental Cooperation Montreal. Quebec. 98 p.

Miller, H. L. 1963. The behavior of the pumpkinseed sunfish, Lepomis gibbous (Linneaus), with notes on the behavior of other species of Lepomis and the pigmy sunfish, Elassoma evergladei. Behaviour 22:88-150.

Neves, A. M. B. y M. L. Ruffino.1998. Aspectos reproductivos do acarí bodó, Liposarcus pardalis (Pisces, Siliformes, Locariidae) (Castelnau,1855) no Médio de Amazonas. Boletim do Museu Paraense Emilio Goeldi. Série Antropologica 14:77-94.

Nikolsky, G. 1963. The ecology of fishes. Academic, New York. $352 \mathrm{p}$.

Ocaña, D. y A. Lot. 1996. Estudio de la vegetación acuática vascular del sistema fluvio-laguna-deltáico del río Palizada, en Campeche, México. Anales del Instituto de Biología. Universidad Nacional Autónoma de México, Serie Botánica 67:303-327.

Páez-Osuna, F., D. Valdez-Lozano, H. M. Alexander y H. Fernández-Pérez. 1987. Trace metals in the fluvial system of Terminos Lagoon, Mexico. Marine Pollution Bulletin 18:294 -297.

Page, L. y R. H. Robins. 2006. Identification of sailfin catfishes (Teleostei:Locariidae) in Southeastern Asia. The Raffles Bulletin of Zoology 54:455-457.

Pauly, D. 1984. Fish population dynamics in tropical waters: A manual for use with programmable calculators. Studies and Reviews 8. International Center for Living Aquatic Resources Management (ICLARM), Makati City, Manila. $325 \mathrm{p}$.

Petry, A. C. y U. H. Schulz. 2000. Ritmo de alimentação de juvenis de Loricariichthys anus (Siluriformes, Loricariidae) da Lagoa dos Quadros, RS, Brasil. Iheringia, Série Zoologia 89:171-176.

Power, M. E. 1984a. Depth distribution of armored catfish: predator induced resource avoidance. Ecology 65:523-528.

Power, M. E. 1984b. Habitat quality and the distribution of algae-grazing catfish in a Panamanian stream. Journal of Animal Ecology 53:357-374.

Power, M. E. 1990. Resource enhancement by indirect effects of glazers: armored catfish, algae and sediment. Ecology 71:897-904.

Rahim, K. A. A., S. K. Daud, S. S. Siraj, A. Arshad, Y. Esa y E. R. Ibrahim. 2009. Freshwater fish diversity and composition in Batang Kerang floodplain, Balai Ringin, Sarawak. Pertanika. Journal of Tropical Agriculture Science 32:7-16.

Ramos-Miranda, J., D. Flores-Hernández, L. A. Ayala-Pérez, J. Rendón-Von Hosten, G. Villalobos-Zapata y A. Sosa-López. 2006. Atlas hidrológico e ictiológico de la laguna de Términos. Centro de Ecología, Pesquerías y Oceanografía del Golfo de México (EPOMEX)-Universidad Autónoma de Campeche, Campeche, Campeche. 173 p.

Rendón-Von Osten, J. M. Memije-Canepa y N. A. Ek-Moo. 2005. Plaguicidas orgánicos persistentes (POPs) en sedimentos de la costa sur de Campeche, México. In Golfo de México. Contaminación e impacto ambiental: diagnóstico y tendencias, segunda edición, A. Botello, J. Rendón-Von Osten, G. Gold-Bouchout y C. AgrazHernández (eds.). Universidad Autónoma de Campeche/ Universidad Autónoma Nacional de México/ Instituto Nacional de Ecología, México, D.F. p. 249-260.

Ribeiro, M. C. L. B. 1983. As migrações dos jaraquis (Pisces, Prochilodontidae) no Rio Negro, Amazonas, Brasil. Dissertação de mestrado, Instituto Nacional de Pesquisas da Amazonia - Fundação Universidade do Amazonas, Aleixo, Manaus. 192 p.

Ricker, W. E. 1975. Computation and interpretation on biological statistics of fish population. Bulletin of Fisheries Research Board of Canada 191:1-392.

Saint-Paul, U., J. Zuanon, M. A. Villacorta Correa, M. García, N. N. Fabré, U. Berger y W. J. Junk. 2000. Fish communities in central Amazonian white and blackwater floodplains. Environmental Biology of Fishes 57: 235-250.

Samat, A., M. N. Shukor, A. G. Mazlan, A. Arshad y M. Y. Fatimah. 2008. Length-weight relationship and condition factor of Pterygoplichthys pardalis (Pisces: Loricariidae) in Malaysia Peninsula. Research Journal of Fisheries and Hydrobiology 3:48-53.

Santana-Hernández, H., R. Macías Z. y A.L. Vidaurri. 1996. Relación entre la abundancia de peces de pico y la temperatura del agua en el Pacífico mexicano. Ciencia Pesquera 13:62-65.

Schulz, U. H. y C. Leuchtenberger. 2006. Activity patterns of South American silver catfish (Rhamdia quelen). Brazilian Journal of Biology 66:565-574.

Sokal, R. R., Rohlf, F. J. 1987. Introduction to biostatistics, segunda edición. Freeman, New York. 363 p.

Tan, B. C. y K. S. Tan. 2003. Singapore. In Invasive alien species in South-Southeast Asia. National reports and directory of resources, N. Pallewatta, J. K. Reaser y A. T. Gutiérrez (eds.). Global Invasive Species Programme, Cape Town, South Africa. p. 85-90.

Tesch, W. 1971. Age and growth. In Methods for assessments of fish production in freshwaters, W. E. Ricker (ed.). International Biological Programme, Oxford. p. 97-130.

Toledo, A. 2003. Ríos, costas, mares. Hacia un análisis integrado de las regiones hidrológicas de México. Instituto Nacional de Ecología, México, D.F. 117 p.

Wakida-Kusunoki, A. T. y L. E. Amador-del Ángel. 2008. Nuevos registros de los plecos Pterygoplichthys pardalis (Castelnau, 1855) y P. disjunctivus (Weber 1991) en el Sureste de México. Hidrobiológica 18:251-255.

Wakida-Kusunoki, A. T., R. Ruiz-Carus, y L. E. Amador-del 
Ángel. 2007. Amazon sailfin catfish, Pterygoplichthys pardalis (Castelnau, 1855) (Loriicaridae), another exotic species established in Southeastern Mexico. The Southwestern Naturalist 52:141-144.

Weber, C. 2003. Subfamily Hypostominae (armored catfishes). In Check list of the freshwater fishes of South and Central America, R. E. Reis, S. O. Kullander y C. J. Ferraris, Jr. (eds.). EDIPUCRS, Porto Alegre, Rio Grande do Sul. $\mathrm{Xi}+729 \mathrm{p}$.

Weimer, R. C. 1996. Estadística, segunda edición. CECSA, México, D.F. 839 p.
Welcomme, R. L. 1979. Fisheries ecology of floodplain rivers. Longman, London. 317 p.

Welcomme, R. L. 1985. River fisheries. FAO Fisheries Technical Paper 262:1-330.

Winemiller, K. O. 1987. Feeding and reproductive biology of the currito, Hoplosternum littorale, in the Venezuelan llanos with comments on the possible function of the enlarged male pectoral spines. Environmental Biology of Fishes 20:219-227.

Zar, J. H. 1996. Biostatistical analysis,,tercera edición. Prentice Hall, Upper Saddle River, New Jersey. 960 p. 